\title{
An Update- The Role of Nutrients Crucial in the Infertility of Couples- New Insights for the Effects of Iodine, Selenium, Omega 3 Fatty Acids and Magnesium
}

\author{
Heverton Alves Peres ${ }^{1 *}$, Maria Cristina Freitas Foss ${ }^{1}$, Leonardo Régis Leira Pereira ${ }^{2}$ and Carlos \\ Manuel Viana ${ }^{3}$ \\ ${ }^{1}$ Department of Internal Medicine, Ribeirão Preto Medical School, University of Sao Paulo, Ribeirão Preto, Sao Paulo, Brazil \\ ${ }^{2}$ Department of Pharmaceutical Sciences, School of Pharmaceutical Sciences of Ribeirao Preto, University of Sao Paulo, Ribeirao \\ Preto, Sao Paulo, Brazil \\ ${ }^{3}$ Viana Health Center, Aruba, Chine, Brazil
}

Received: October 05, 2017; Accepted: November 29, 2017; Published: December 07, 2017

*Corresponding author: Heverton Alves Peres, pharmacist and doctorate in medical clinical in Faculty of Medicine of Ribeirao Preto-SP, Brazil, Tel: 55 16-9215-4036, E-mail: haperes@usp.br

\begin{abstract}
Infertility is a global public health issue that affects more 186 million people worldwide and remains a woman's social burden, although male infertility contributes to more than half of all causes of global childlessness. Factors as the age, obesity, weight, insulin resistance, psychological stress, toxins, infections, environmental and occupational exposures, electromagnetic pollution and nutritional deficiency can have effects on fertility. Nutrients that are crucial in successful fecundity include: iodine, selenium, omega 3 fatty acids and magnesium. Unfortunately, the importance of these supplements in fertility are not known or forgotten by most doctors, principally fertility specialists. Thus, this mini-review's objective is to elucidate the efficacious role provide by iodine, selenium, omega 3 fatty acids and magnesium in fecundity.
\end{abstract}

\section{Introduction}

Barrenness, not producing or incapable of producing offspring when desired after twelve months, is a global public health issue affecting more than 186 million people worldwide. Not producing offspring remains a woman's social burden; although, male infertility contributes to more than half of all causes of global infertility. It is estimated that 8 to $12 \%$ of reproductiveage couples worldwide are affected by fecundity problems and in some areas reaching $30 \%$ [1]. In Brazil, the infertility rate affects $20 \%$ of couples and fecundity has been decreasing every year. Brazilian fertility rate is now 1.76 newborns per couple [2]. Factors that can affect fecundity include: age, obesity, weight, insulin resistance, psychological stress, toxins, infections, environmental and occupational exposures, electromagnetic pollution and nutritional deficiency $[3,4]$.

Underpinning most disease, usually, exists contributing nutritional deficiencies. Disease does not thrive in a heathy body.
Pathology in most sick patients presents concurrently with a constellation of metabolic deficiencies. Nutrients that are crucial in successful fecundity include: iodine, selenium, omega 3 fatty acids and magnesium. Unfortunately, the importance of these supplements in fertility are not known or forgotten by most doctors, principally fertility specialists. Additionally, in Brazil and other countries the focus of fertility focuses on women, while men's health status is overlooked.

Our hypothesis is that specific dietary supplements can improve fertility. Specifically, we hypostasize, in women, dietary iodine can ameliorate Poly-Cystic Ovarian Syndrome (PCOS) while regulating thyroid function and magnesium supplementation can reduce resistance insulin. In men, selenium and omega 3 fatty acids can improve sperm mobility, increasing fertilization chances. Thus, this mini-review's objective is to elucidate the efficacious role provide by iodine, selenium, omega 3 fatty acids and magnesium in fecundity. Further, this paper's aim is to provide relevant information to support a positive decision for doctors and health professionals to co-prescribe these supplements to treat fecundity problems.

\section{Method}

In this review, we focused in systematic review, randomized clinical trial and observational studies published in the last seven years. Thus, these studies are essentials for advancing our knowledge about role of dietary supplementary use in the prevention and fight against the diseases, mainly infertility. Our aim is to address the paucity in medical literature on the positive fertility effects provided through dietary supplementation. 


\section{Iodine}

Regrettably, iodine is an essential nutrient barely understood by the current model medical; due to lack of knowledge, and prescription fear. Recently, our research group published a review to clarify some doubts and encourage doctors and health professionals to prescribe iodine for prevention and treatment of various medical conditions. The halogens are a group of five chemically related elements: fluorine $(\mathrm{F})$, chlorine $(\mathrm{Cl})$, bromine $(\mathrm{Br})$, iodine (I), and astatine (At). The halogens fluoride, chloride and bromine displace iodine in its receptors [5-48].

Today, worldwide, various pharmaceuticals containing bromides, fluorine and/or chlorine and ingested bromide from grain products breads, inhibit iodine docking in its receptor. Thus, displacing bromides reduce in availability of iodine in the organs that that actively concentrate iodine including the thyroid, stomach mucosa, mammary glands, salivary glands, thymus, choroid plexus, kidney, joints, arteries, and bones.

A purple-black metallic solid in standard condition, iodine is the heaviest and most stable of the halogens. The human body stores around 1,500 mg iodine. A crucial micronutrient and a component of the thyroid hormones, the thyroid stores only 50 mg iodine to convert into thyroxine (T4) and triiodothyronine (T3). Additionally, iodine plays a crucial role in the development of various organs [6-7]. Almost all cells in the human body use iodine that is adsorbed in glandular tissues such as: the salivary glands, breasts, parotids, pancreas, prostate, lachrymal glands and gastric mucosa. In the immunological system, the white blood cells need of iodine to fight against infectious agents.

Worldwide, iodine deficiency affects approximately $95 \%$ of the population. An ongoing public health problem, in Brazil, as well as many other countries, exists an iodine deficient population. The National Health and Nutrition Examination Survey (NHANES) disclosed that in the last 30 years, there is a $50 \%$ decreased in the iodine ingestion [49]. Iodine consumption levels are higher in The People's Republic of China and Japan through the consumption of seaweed. Compared with women worldwide Chinese and Japanese women have lower rates of perinatal mortality; attributed to iodine levels in consumed seaweed [5051]. A systematic review completed in Brazil showed that only a few studies have been undertaken to establish Iodine Deficiency Disorder (IDD). Prevalence of IDD has only been established in the Southeastern to South Brazilian regions. However, reliability of these studies is being questioned because of the complexity due to individual study bias and the very high heterogeneity [52]. Iodine deficiency results in endemic goiter, endemic mental retardation, increased prenatal death and infant mortality, and decreased fertility $[8,9]$. In this baseline, we present the medical iodophobia regarding iodine supplementation, iodine deficiency and a need to change medical standards and procedures regarding iodine are public health system challenges.

Hypothyroidism is a disease common sometimes undiagnosed in many women whose etiology, among others, is caused by iodine deficiency. Hypothyroid has a major impact on fertility and increases miscarriage risk. Iodine deficiency negatively effects folliculogenesis in the maturation of the ovarian follicle leading to Polycystic Ovary Syndrome (PCOS). When ovarian follicles do not mature, the ovaries cannot release eggs; and concomitant with the hyperproduction of male hormone, testosterone [10]. Although the exact cause of PCOS is yet unidentified some factors are recognized: insulin resistance, obesity, heredity, iodine deficiency, low-grade inflammation and anything that disrupts hormone disruption can influence the onset of PCOS [3,5-11]. When the iodine levels are deficient in the thyroid, certainly there must be a deficiency in the ovaries. Thus, sustaining sufficient iodine levels promotes ovarian health through successful folliculogenesis.

Glucose adsorbs into cells by insulin successful docking in its receptor, releasing Glucose Transporter Type 4 (GLUT4) that pulls glucose into the cell. Once inside the cell, glucose is processed through the Krebs cycle, in the mitochondria to produce adenine triphosphate (ATP), processing energy. Many exogenous factors prevent insulin from docking in its receptors, called insulin resistance. The beta cells in the islets of Langerhans in the pancreas continue making insulin, but the insulin does not dock. Serum glucose rises and the pancreas increases insulin production is not available to the cells to convert glucose to ATP. Insulin resistance is the preclinical stage of type II diabetes. An exogenous factor in insulin resistance is iodine deficiency. Jorge Flechas, MD, MPH, showed iodine attaches to insulin receptors, increasing insulin sensitivity. Additionally, since iodine is a cofactor at the cellular receptor for all hormones in the human body, including insulin and glucagon, iodine deficiency produces systemic pathology. Deficiency of both hormones through iodine deficiency presents with hyperglycemia or hypoglycemia. A diet high in carbohydrates (white sugar, white flour, and simple carbohydrates) combined with iodine deficiency exacerbates symptoms because of widely fluctuating blood sugar levels. This iodine deficiency pathology, unfortunately, it is not widely known by gynecologists and health professionals.

Our aim is to rectify the medical iodophobia beginning in 1948 when Wolff and Ckaikoff reported that after a certain iodine supplemented, rat thyroids do not adsorb most iodine and extrapolated their findings to humans. They construed iodine destroys the thyroid. However, the researchers neither failed to measure associated thyroid hormones nor realizing the iodine saturation phenomenon. Later, Wolff seated as the director of the National Institute of Health (NIH) he used his influence with medical schools to teach not to prescribe iodine. Wolff's iodophobia was adopted in all nutrition and medicine textbooks including endocrinology. What destroys the thyroid causing hypothyroidism is organic iodine compound used as an X-ray contrast medium used to enhance the visibility of internal structures in X-ray-based imaging techniques such as Computed Tomography (CT), radiography, and fluoroscopy. Dietary supplement, inorganic iodine, is not toxic to the thyroid, is utilized by human tissue and can be used efficaciously to treat and prevent various diseases $[5,12]$. 
Iodine deficiency is linked to ovarian cysts that hinder fertility and increase the risk of ovarian cancer. Iodine deficiency further can develop nodules and form fibrosis that can progress to cancer of thyroid, uterus, and breasts. According with National Health and Nutrition Examination Survey (NHANES), in the last 30 years the iodine intake has decreased 50\% [13]. The People's Republic of China and Japan has the lowest perinatal mortality rates because women ingest a copious amount of iodine rich seaweed $[14,15]$. Currently, there are various systematic reviews encouraging the safe use iodine in the dosages of $250 \mu \mathrm{g} /$ day by lactating women and $200 \mu \mathrm{g} /$ day during pregnancy; unfortunately, only a scarce number of doctors co-prescribe iodine [5]. Infertility treated with iodine supplementation should include selenium supplementation, dosed at 200-400 $\mu \mathrm{g} /$ day to avoiding hydrogen peroxide formation by tyrosine oxidase. Selenium supplementation stimulates the formation of glutathione peroxidase that neutralizes hydrogen peroxide. Additionally, supplementation with vitamin C dosed 2,000$5,000 \mathrm{mg} /$ day and Himalayan salt (one spoonful diluted in oneliter water/day) provide antioxidant support. These additional supplements also stimulate the integral membrane protein, symporter that is involved in the transport of many differing types of molecules across the cell membrane, specially the NIS (Sodium/Iodine Symporter) that stimulates TSH to better uptake iodine and sodium.

\section{Selenium}

Selenium (Se) is a crucial trace element for more than two dozen protein that includes a selenocysteine (Sec, U, Se-Cys) amino acid residue, selenoproteins that play an essential role in reproduction, egg production, DNA synthesis, and thyroid hormone metabolism. Selenoproteins prevent free radicals cellular damage and also helps support the immune system. Se also protects against miscarriage and birth defects and currently, Se supplementation is recommended as public health policy in areas where soils are deficient in selenium [16]. Problems with iodine and selenium deficiencies affect almost half the world population, especially, due to low concentration of soil micronutrients, or not possessing the optimal available soil $\mathrm{pH}$ within this 6.5 to $7.5 \mathrm{pH}$, where nutrients are optimally available to plants [17]. In Brazil as in other countries, to date, current population data of Selenium ingestion is still scarce; however, it has been verified that various Brazilians agricultural products have low Selenium levels [18-20].

Some studies address the relationship between selenium consumption and fertility because of its significant role in the functioning of the natural reproductive system $[20,21]$. Reduction of fertility rates in women has been linked to deficient selenium body saturation; yet, selenium's role in this process remains unknown. One proposition is that selenium may neutralize, eliminate and prevent the synthesis of Reactive Oxygen Species (ROS) functioning as a co-factor of antioxidative enzymes [22, 23]. Increase in ROS concentrations negatively affects immature ova, oocytes in the first meiotic divisions, but selenium inhibits the ROS negative effects [24, 25]. Low selenium concentration has been observed in follicular fluid in women with unexplained infertility when compared to women infertility due to other factors. Authors of these studies suggest that activity of glutathione peroxidade (GPx) in the follicular microenvironment may play an essential role in the fertilization and gametogenesis [26]. Selenium deficiencies may lead to miscarriages, damaging the nervous and immune system of fetuses and produce gestational complications. Additionally, low concentration of selenium in blood serum has been associated with newborn low birth weight [20].

Sperm counts among men have more than halved during the last 40 years, and the latest findings reveal that the concentration of male sperm ejaculate in western countries has continued to fall an average of $1.4 \%$ a year, leading to an overall drop of just over $52 \%$. Low sperm counts might also be an indicator of nutritional deficiency, body weight, lack of physical activity and smoking [31]. Selenium deficiency also produces infertility in men reducing the quality of semen and low sperm motility. Antioxidant therapy provided by selenium, glutathione, vitamins $\mathrm{C}$ and $\mathrm{E}$, zinc, folic acid, $\mathrm{N}$-acetylcysteine and Coenzyme-Q10 may reduce oxidative stress induced sperm damage increasing live birth rates [27-29]. Some clinical trials encourage using selenium combined with other antioxidants to treat male infertility. One systematic review by the Cochrane group reported that antioxidant may provide benefit for reduced fertility with prolonged time of unwanted non-conception, sub fertile women $[21,30]$. However, even with the literature being prodigious in demonstrating the benefits of selenium in the treatment of infertile couples, unfortunately, only a few doctors in the Brazil and elsewhere co-prescribe this nutrient.

\section{Omega 3}

Some omega- 3 (n-3) and omega- 6 (n-6) fatty acids neither be synthesized by animals nor humans and need to be consumed. Accordingly, linolenic acid (C18:3 n-3) and linoleic acid (C18:2 n-6) are critical in numerous somatic processes, including: general growth, development of the brain, eyes and reproductive system. Mammalian spermatozoa have a high proportion of Polyunsaturated Fatty Acids (PUFA), especially palmitic acid (C16:0) and docosahexanoic acid (DHA, C22:6n-3) both, besides possessing wide-range of health benefits, play a pivotal role in fertilization. However, overconsumption of soybean oil, linoleic acid (omega-6), has been linked with decreased eicosapentaenoic acid tissue concentration (EPA; also, icosapentaenoic acid, an omega-3 fatty acid) and docosahexaenoic acid (DHA) in the United States during the 20th century and may explain the decrease in male fertility as a pivotal health problem worldwide [34]. Consequently, the increased intake of omega- 6 fatty acid and the decrease of omega- 3 fatty acid contribute to a large increase in the majority of developed countries in the unhealthy omega-6/ omega-3 ratio of 20:1. Additionally, corporate agribusiness and modern agriculture practices produces a Western diet containing low levels of omega- 3 and high levels of omega- 6 . This nutritional 
shift is linked to a significant increase in the prevalence of weight gain and obesity factors that contribute to infertility [53].

Currently, medical concerns exist because low DHA levels in human spermatozoa may be linked infertility. Exacerbating the problem, elevated Tran's fatty or saturated fat concentration decrease DHA in spermatozoa [32, 33]. Clinical trial using omega-3 supplementation in men with deficient sperm is limited. Healthy human sperm anatomy, the tail and head have higher DHA levels and the sperm head contains more DHA. It has been shown supplementation with omega-3 for more than four weeks improves the sperm analysis results. Differences in dose-dependent results and time-dependent values may explain failure experienced in various experiments. Ingested dietary plant omega- 3 sources, such as walnuts that contain antioxidants (75 g per day for 12 weeks) improve sperm vitality, morphology and motility [35]. Both omega- 3 and omega- 6 fatty acids have been shown to improve low sperm concentration in oligospermic men, significantly reduces ROS and increase acrosome reaction potential, allowing sperm to penetrate the zona pellucida and fuse with the oocyte membrane [36]. Fish oil supplementation with EPA and DHA (1.84 g per day for 32 weeks) in men with low sperm count, poor sperm mobility, and abnormal sperm shape, oligoasthenoteratozoospermia showed improved sperm concentration and total sperm count [37]. Additionally, both EPA and DHA provide an essential role in various enzymes such as Superoxide Dismutase (SOD) that protects the spermatozoa against peroxidative damage. Undeniably, antioxidant activity of semen is improved with omega- 3 as reported and some mechanisms about the effect of EPA and DHA on spermatogenesis were presented, although the mechanisms are not clearly known.

Painful periods, or menstrual cramps, dysmenorrhea is common in infertile women due to disbalance between antiinflammatory, vasodilating, eicosanoids derived from omega-3 fatty acids and proinflammatory, vasoconstrictor eicosanoids derived from omega-6 fatty acids. Dysmenorrhea symptoms can be ameliorated when supplementation of omega- 3 fatty acids increases. Omega-3 fatty acids can increase the ratio of thromboxane to prostacyclin helping pregnancy by increasing uterine blood flow [38]. Recently, data of a review reinforce that low intake Trans fatty acid and higher intake of long-chain omega-3 fatty acids may be beneficial for female fertility [57].

\section{Magnesium}

Magnesium is one of most abundant mineral in the human body. Magnesium ions regulate over 300 biochemical reactions in the body through their role as enzyme co-factors. They also play a vital role in the reactions that generate and use ATP, the fundamental unit of energy within the body's cells. Positive health is dependent on adequate magnesium levels since this mineral is essential for regulating blood pressure, maintaining a healthy heart rhythm, helping control blood sugar levels and keeping the muscles resilient. Data from the World Health Organization (WHO) estimate that three quarters of North Americans do not consume the recommended daily intake of magnesium [54]. A
French study presented that $80 \%$ of women and $70 \%$ of men are magnesium deficient [55]. In Finland when magnesium was supplemented in table salt, the death rates due to chronic heart disease fell to a better worldwide ranking of 10th [56].The RDA (Recommended Dietary Allowances) for magnesium in men is $420 \mathrm{mg} /$ day and for women $320 \mathrm{mg} /$ day, however the average daily consumption has been much lower than the recommended amounts, leading to a myriad of pathology, including infertility $[39,40]$.

Magnesium is essential in various developmental stages of sperm production and function, including governing the rate limiting steps in DNA synthesis and mitosis. Magnesium deficit is associated with a wide range of complications of female and male reproductive systems. Deficiency increases infertility, and the risk of miscarriage, pre-term birth and low birthweight babies. The essential requirement of magnesium for sex hormone production and function underlies the importance of magnesium in infertility. It is known that estrogen function is a magnesium-dependent process and magnesium controls FSH (hormone stimulating the ovaries) binding to receptors on the ovary. It is also known that magnesium is important in governing the rate limiting steps in protein synthesis and cell division. Complications in the reproductive system of men and women are associated with magnesium deficiency, where deficiency increases infertility, the risk of miscarriage and low weight babies [41-43]. Magnesium is essential for sex hormone production and infertility and it's is known that estrogen receptor is a magnesium-dependent process besides of modulates FSH binding to receptors on the ovary [4446]. Additionally, magnesium deficiency has been associated with increased smooth muscle cell tone that may reduce the obstruction of an otherwise normal, open fallopian tube. Further, a study about magnesium supplementation on infertile women showed that all women have low Red Blood Cell (RBC) magnesium levels, although not all women returned to normal even when give $600 \mathrm{mg}$ daily of magnesium for four months. To achieve optimal RBC magnesium levels, necessitates the oral dosing of selenium 200 mcg daily. After normalizing their RBC magnesium levels, all infertile women delivered normal healthy babies [47].

\section{Conclusions}

The current medical paradigm need urgent attitude changes about prescribing of nutrients for infertility. We confirmed the hypothesis that iodine, selenium, omega 3 fatty acids and magnesium are nutrients crucial for successful fecundity of couples. In the women, iodine supplementation ameliorates PCOS and regulating thyroid function while magnesium supplementation can reduce resistance insulin. Gynecologists and fertility specialists should not neglect the health of men and selenium and omega 3 fatty acids supplementation can improve sperm mobility increasing fertilization chances. Recommendations for clinical trials with infertile couples taking iodine, selenium, omega 3 fatty acids and magnesium in the correct dosages including longer follow up of couples in the period of supplementation. 


\section{References}

1. Inhorn MC, Patrizio P. (2015). Infertility around the globe: new thinking on gender, reproductive technologies and global movements in the 21st century. Hum Reprod update. 21(4):411-426. Doi: 10.1093/ humupd/dmv016

2. http://www.indexmundi.com/brazil/total_fertility_rate.html, accessed in the 2 August 2017.

3. Peres HA, Pereira LRL, Foss MCF. Toxins, Malnutrition, Stress, Infections and Electromagnetic Pollution: Looking about New Perspectives in Development of Diseases. J Nutr Food Sci. 6:558. doi: 10.4172/21559600.1000558

4. Sharma R, Biedenharn KR, Fedor JM, Agarwal A. Lifestyle factors and reproductive health: taking control of your fertility. Reprod Biol Endocrinol. 2013;11:66. doi: 10.1186/1477-7827-11-66

5. Peres HA. Iodine, Ingesting or not? J Nutr Disorders Ther. 2017;7:206. doi:10.4172/2161-0509.1000206

6. Zimmermann MB. The role of iodine in human growth and development. Semin Cell Dev Biol. 2011;22(6):645-652. doi: 10.1016/j.semcdb.2011.07.009

7. Andersson M, Karumbunathan V,Zimmermann MB. Global iodine status in 2011 and trends over the past decade. J Nutr. 2012;142(4):744-750. doi: $10.3945 /$ jn.111.149393

8. FAO/WHO. 2nd ed. Geneva: World Health Organization; 2005. Vitamin and mineral requirements in human nutrition

9. Nordic Nutrition Recommendations 2004. 4th ed. Denmark: Arhus; 2005. Integrating nutrition and physical activity. Nordic Council of Ministers.

10. Gandar R, Spizzo M, Collin D. Diagnosis and treatment of polycystic ovary syndrome. Journal de gynecologie, obstetrique et biologie de la reproduction. 28(6):510-518.

11. Shorakae S, Teede H, de Courten B, Lambert G, Boyle J, Moran LJ. The emerging role of chronic low-grade inflammation in the pathophysiology of polycystic ovary syndrome. Semin Reprod Med. 2015;33(4):257-269. doi: 10.1055/s-0035-1556568

12. Wolff J, Chaikoff IL. Plasma inorganic iodide as a homeostatic regulator of thyroid function. Journal of Biological Chemistry. 1948;174:555564.

13. Caldwell KL, Miller GA, Wang RY, Jain RB, Jones RL. Iodine status of the US population, National Health and Nutrition Examination Survey 2003-2004. Thyroid. 2008;18(11):1207-1214. doi: 10.1089/ thy. 2008.0161

14. DeLong GR, Leslie PW, Wang SH, Jiang XM, Zhang ML, Abdul-Rakeman $\mathrm{M}$, et al. Effect on infant mortality of iodination of irrigation water in a severely iodine-deficient area of China. Lancet. 1997;350(9080): 771773.

15.Zava TT, Zava DT. Assessment of Japanese iodine intake based on seaweed consumption in Japan: a literature-based analysis. Thyroid res. 2011;4:14. doi: 10.1186/1756-6614-4-14

16.https://ods.od.nih.gov/factsheets/Selenium-HealthProfessional/, accessed in the 17 August 2017.

17. Dos Reis AR, El Ramady H, Santos EF, Gratão PL, Schomburg, L. Overview of Selenium Deficiency and Toxicity Worldwide: Affected Areas, Selenium-Related Health Issues, and Case Studies. In Selenium in plants. Springer International Publishing. 2017;209-230.
18. Nogueira Tar, Alleoni Lrf, He Z, Villanueva Fca, Poggere Gc, Abreu Junior Ch. Natural Contents And Quality Reference Value For Selenium In Soils Of The State Of São Paulo. In: Xxxiv Brazilian Congress On Science Of The Soil. Annals. 2013;Florianópolis. Cd-Rom.

19. Haug A, Graham RD, Christophersen OA, Lyons GH. How to use the world's scarce selenium resources efficiently to increase the selenium concentration in food. Microb Ecol Health Dis. 2007;19(4):209-228. doi: 10.1080/08910600701698986

20. Pieczyńska J, Grajeta H. The role of selenium in human conception and pregnancy. J Trace Elem Med Biol. 2015;29:31-38. doi: 10.1016/j. jtemb.2014.07.003

21. Showell MG, Brown J, Clarke J, Hart RJ. Antioxidants for female subfertility. Cochrane Database Syst Rev. 2013;5(8):CD007807. doi: 10.1002/14651858.CD007807

22. Ruder EH, Hartman TJ, Blumberg J, Goldman MB. Oxidative stress and antioxidants: exposure and impact on female fertility. Hum Reprod Update.2008;14(4):345-357. doi: 10.1093/humupd/dmn011

23. Ruder EH, Hartman TJ, Goldman MB. Impact of oxidative stress on female fertility. Curr Opin Obstet Gynecol. 2009;21(3):219-222.

24. Takami M, Preston SL, Toyloy VA, Behrman HR. Antioxidants reversibly inhibit the spontaneous resumption of meiosis. Am J Physiol. 1999;276(4):E684-E688.

25. Takami M, Preston SL, Behrman HR. Eicosatetraynoic and eicosatriynoic acids, lipoxygenase inhibitors, block meiosis via antioxidant action. Am J Physiol Cell Physiol. 2000;278(4):C646-C650.

26. Paszkowski T, Traub AI, Robinson SY, McMaster D. Selenium dependent glutathione peroxidase activity in human follicular fluid. Clin Chim Acta. 1995;236(2):173-180.

27. Majzoub A, Agarwal A. Antioxidant therapy in idiopathic oligoasthenoteratozoospermia. Indian J Urol. 2017;33(3):207-214. doi: 10.4103/iju.IJU_15_17

28. Ahmadi S, Bashiri R, Ghadiri-Anari A, Nadjarzadeh A. Antioxidant supplements and semen parameters: An evidence based review. Int J Reprod Biomed. 2016;14(12):729-736.

29. Mora-Esteves C, Shin D. Nutrient supplementation: improving male fertility fourfold. Semin Reprod Med. 2013;31(4):293-300. doi: 10.1055/s-0033-1345277

30. Moslemi MK, Tavanbakhsh S. Selenium-vitamin E supplementation in infertile men: effects on semen parameters and pregnancy rate. Int J Gen Med. 2011;4:99-104. doi: 10.2147/IJGM.S16275

31. Levine H, Jørgensen N, Martino-Andrade A, Mendiola J, WekslerDerri D, Mindlis I, et al. Temporal trends in sperm count: a systematic review and meta-regression analysis. Human Reproduction Update. 2017;23(6):646-659.

32. Esmaeili V, Shahverdi AH, Moghadasian MH, Alizadeh AR. Dietary fatty acids affect semen quality: a review. Andrology. 2015;3(3):450-461. doi: 10.1111/andr.12024

33. Martínez-Soto JC, Landeras J, Gadea J. Spermatozoa and seminal plasma fatty acids as predictors of cryopreservation success. Andrology . 2013;1(3):365-375. doi: 10.1111/j.2047-2927.2012.00040.x

34. Blasbalg TL, Hibbeln JR, Ramsden CE, Majchrzak SF, Rawlings RR. Changes in consumption of omega- 3 and omega- 6 fatty acids in the United States during the 20th century. Am J Clin Nutr. 2011;93(5):950962. doi: $10.3945 /$ ajcn. 110.006643 
35. Robbins WA, Xun L, FitzGerald LZ, Esguerra S, Henning SM, Carpenter CL. Walnuts improve semen quality in men consuming a Westernstyle diet: randomized control dietary intervention trial. Bio Reprod. 2012;87(4):101. doi: 10.1095/biolreprod.112.101634

36. Safarinejad MR, Safarinejad S. The roles of omega- 3 and omega- 6 fatty acids in idiopathic male infertility. Asian J Androl. 2012;14(4):514515. doi: 10.1038/aja.2012.46

37. Safarinejad MR. Effect of omega-3 polyunsaturated fatty acid supplementation on semen profile and enzymatic anti-oxidant capacity of seminal plasma in infertile men with idiopathic oligoasthenoteratospermia: a double-blind, placebo-controlled, randomised study. Andrologia. 2011;43(1): 38-47. doi: 10.1111/j.1439-0272.2009.01013.x

38. Saldeen P, Saldeen T. Women and omega-3 Fatty acids. Obstet Gynecol Surv. 2004;59(10):722-730.

39. Chacko SA, Song Y, Nathan L, Tinker L, De Boer IH, Tylavsky F, et al. Relations of dietary magnesium intake to biomarkers of inflammation and endothelial dysfunction in an ethnically diverse cohort of postmenopausal women. Diabetes care. 2010;33(2):304-310. doi: 10.2337/dc09-1402

40. Ramirez AVG. The importance of magnesium in cardiovascular disease. International Journal of Nutrology. 2016;9(4):242-253.

41. De Ridder G, Gossen D. Magnesium in pregnancy--relationship to prophylaxis and therapy of early contractions and threatening premature birth. Magnesium Res. 1:247.

42. Fehlinger R, Kemnitz C, Dreissig P, Egert M, Seidel K. Prematurity, Latent Tetany And Magnesium-Deficiency-A Retrospective Study With 132 Mothers. Magnesium-Bulletin. 6(2):52-59.

43. Wynn A, Wynn M. Magnesium and other nutrient deficiencies as possible causes of hypertension and low birthweight. Nutr Health. 1988;6(2):69-88. doi: 10.1177/026010608800600201

44. Fukai F, Murayama A. Association and dissociation of estrogen receptor with estrogen receptor-binding factors is regulated by Mg2+. J Biochem. 1984;95(4):1227-1230.

45. Ford KA, LaBarbera AR. Cationic modulation of follicle-stimulating hormone binding to granulosa cell receptor. Biol Reprod. 1987;36(3):643-650.
46. Walker GM. Magnesium and cell cycle control: an update. Magnesium. 1986;5(1):9-23.

47. Howard JM, Davies S, Hunnisett A. Red cell magnesium and glutathione peroxidase in infertile women--effects of oral supplementation with magnesium and selenium. Magnes Res. 1994;7(1):49-57.

48. Mann, Jim Truswell, Stewart. Iodine. Essentials of Human Nutrition. Oxford University Press; 172.

49. Caldwell KL, Miller GA, Wang RY, Jain RB, Jones RL. Iodine status of the US population, National Health and Nutrition Examination Survey 2003-2004. Thyroid. 18(11):1207-1214. doi: 10.1089/thy.2008.0161

50. DeLong GR, Leslie PW, Wang SH, Jiang XM, Zhang ML, Abdul-Rakeman $\mathrm{M}$, et al. Effect on infant mortality of iodination of irrigation water in a severely iodine-deficient area of China. Lancet. 1997;350(9080):771773.

51.Zava TT, Zava DT. Assessment of Japanese iodine intake based on seaweed consumption in Japan: a literature-based analysis. Thyroid res. 2011;4:14. doi: 10.1186/1756-6614-4-14

52. Campos RDO, Barreto IDS, Maia LRDJ, Rebouças SCL, Cerqueira TL, et al. Iodine nutritional status in Brazil: a meta-analysis of all studies performed in the country pinpoints to an insufficient evaluation and heterogeneity. Arch Endocrinol Metab. 2015; 59(1):13-22. doi: 10.1590/2359-3997000000004

53. Simopoulos AP. An increase in the omega-6/omega-3 fatty acid ratio increases the risk for obesity. Nutrients. 2016;8(3):128. doi: 10.3390/ nu8030128

54.World Health Organization. Calcium and Magnesium in Drinking Water: Public health significance. Geneva: World Health Organization Press; 2009.

55. Theophanides T, Anastassopoulou J. (1997). Magnesium: Current Status and New Developments: Theoretical, Biological and Medical Aspects. Springer Science \& Business Media.

56. Seelig, MS, Rosanoff A. (2003). The magnesium factor. Penguin.

57. Gaskins AJ, Chavarro JE. Diet and fertility a review. Am J Obstet Gynecol. 2017;pii: S0002-9378(17)30945-6. doi: 10.1016/j. ajog.2017.08.010 